\title{
Diffusion tensor imaging and ventricle volume quantification in patients with chronic shunt-treated hydrocephalus: a matched case-control study
}

\author{
Kristy Tan, PhD, ${ }^{1}$ Avital Meiri, BA, ${ }^{1}$ Wenzhu B. Mowrey, PhD, ${ }^{2}$ Rick Abbott, MD, ${ }^{3}$ \\ James T. Goodrich, MD, PhD, ${ }^{3}$ Adam L. Sandler, MD, ${ }^{3}$ Asif K. Suri, MD, PhD, ${ }^{1,5}$ \\ Michael L. Lipton, MD, PhD, ${ }^{1,4-6}$ and Mark E. Wagshul, PhD',7
}

1Department of Radiology, Gruss Magnetic Resonance Research Center, and Departments of ${ }^{2}$ Epidemiology and Population Health, ${ }^{4}$ Neuroscience, ${ }^{6}$ Psychiatry and Behavioral Sciences, and ${ }^{7}$ Physiology and Biophysics, Albert Einstein College of Medicine; ' ${ }^{D}$ epartment of Neurological Surgery, Children's Hospital at Montefiore; and ${ }^{5}$ Department of Radiology, Montefiore Medical Center, Bronx, New York

OBJECTIVE The object of this study was to use diffusion tensor imaging (DTI) and tract-based spatial statistics (TBSS) to characterize the long-term effects of hydrocephalus and shunting on white matter integrity and to investigate the relationship of ventricular size and alterations in white matter integrity with headache and quality-of-life outcome measures. METHODS Patients with shunt-treated hydrocephalus and age- and sex-matched healthy controls were recruited into the study and underwent anatomical and DTI imaging on a 3-T MRI scanner. All patients were clinically stable, had undergone CSF shunt placement before 2 years of age, and had a documented history of complaints of headaches. Outcome was scored based on the Headache Disability Inventory and the Hydrocephalus Outcome Questionnaire. Fractional anisotropy (FA) and other DTI-based measures (axial, radial, and mean diffusivity; AD, RD, and MD, respectively) were extracted in the corpus callosum and internal capsule with manual region-of-interest delineation and in other regions with TBSS. Paired t-tests, corrected with a 5\% false discovery rate, were used to identify regions with significant differences between patients and controls. Within the patient group, linear regression models were used to investigate the relationship between FA or ventricular volume and outcome, as well as the effect of shunt-related covariates.

RESULTS Twenty-one hydrocephalus patients and 21 matched controls completed the study, and their data were used in the final analysis. The authors found significantly lower FA for patients than for controls in 20 of the 48 regions, mostly posterior white matter structures, in periventricular as well as more distal tracts. Of these 20 regions, 17 demonstrated increased RD, while only 5 showed increased MD and 3 showed decreased AD. No areas of increased FA were observed. Higher FA in specific periventricular white matter tracts, tending toward FA in controls, was associated with increased ventricular size, as well as improved clinical outcome.

CONCLUSIONS The study shows that TBSS-based DTI is a sensitive technique for elucidating changes in white matter structures due to hydrocephalus and chronic CSF shunting and provides preliminary evidence that DTI may be a valuable tool for tailoring shunt procedures to monitor ventricular size following shunting and achieve optimal outcome, as well as for guiding the development of alternate therapies for hydrocephalus.

https://thejns.org/doi/abs/10.3171/2017.6.JNS162784

KEY WORDS diffusion tensor imaging; tract-based spatial statistics; pediatric hydrocephalus; headache; quality of life; chronic shunting; diagnostic technique 
$\mathrm{P}$ ATIENTS with chronic shunt-treated hydrocephalus, who have had a CSF shunt in place since their first few years of life, frequently present with symptoms characteristic of shunt failure and increased intracranial pressure (ICP). ${ }^{10,15,51,56}$ This has been reported to occur in up to $50 \%$ of patients ${ }^{17,29,45}$ and is often present even in the absence of overt shunt failure. Patients may suffer from chronic debilitating headaches, and many demonstrate normal to slitlike ventricles. The extreme case has been termed slit ventricle syndrome (SVS), occurring less frequently, in about $1 \%-5 \%$ of patients. ${ }^{3}$ Theories on the pathophysiology of slitlike ventricles and SVS abound, ranging from noncompliant ventricles to overdrainage leading to intermittent shunt obstruction and craniocephalic disproportion. ${ }^{47}$ In all likelihood, the pathophysiology varies from patient to patient, depending on factors such as hydrocephalus etiology, age at first shunt placement, the type of shunt valve used, and shunt revision history.

This variability has led to disagreement in choosing the optimal treatment for alleviating symptoms and restoring normal intracranial dynamics in these patients. However, even in the absence of symptoms suggesting shunt failure, nothing is known about the deleterious effect of chronic CSF shunting on brain tissue. Subclinical ICP elevation, intermittent shunt obstruction leading to stretching or compression of the periventricular white matter (PVWM), and variability in CSF dynamics can all have effects on the brain and may be at least partially responsible for longterm outcome and clinical symptomology.

Diffusion tensor imaging (DTI) is an MRI-based imaging technique that uses measurement of the anisotropic nature of water diffusion in the white matter, primarily caused by preferential diffusion along the axons making up white matter tracts. ${ }^{7,36}$ Changes in white matter integrity are usually quantified by changes in fractional anisotropy (FA), a parameter varying from 0 (e.g., in CSF, where diffusion is not directionally dependent) to 1 (e.g., in intact white matter tract with unidirectional diffusion along the axon). Thus, changes in FA are indicative of white matter integrity and have been used to classify disease severity and response to therapy in a multitude of diseases, such as Alzheimer's disease, mild cognitive impairment, ${ }^{13,14} \mathrm{Par}-$ kinson's disease, ${ }^{39,67}$ and dementia with Lewy bodies. ${ }^{28} \mathrm{In}$ hydrocephalus, DTI has been used to document changes in the PVWM in normal pressure hydrocephalus (NPH), as well as its reversal toward normal following successful shunting., ${ }^{1,5,9,11,50}$ For the most part, these studies have demonstrated reduction of FA in the corpus callosum and elevated FA in the internal capsule extending into the caudal corticospinal tracts. Unfortunately, there have only been a limited number of studies in pediatric hydrocephalus, ${ }^{12,19,22,33,44,55,64}$ with only 1 series involving patients with chronic shunt-treated hydrocephalus (hydrocephalus secondary to spina bifida $\left.{ }^{61}\right)$. In comparison with the effects of slit ventricle or normal ventricular size often seen in chronic shunting, these studies have mostly documented the effect of moderate to severe ventriculomegaly on FA in the PVWM.

In this study, we used DTI to quantify changes in the directionality of water diffusion in PVWM and other more distal white matter tracts in shunt-dependent patients who had undergone shunt placement within the first 2 years of life. Tract-based spatial statistics (TBSS) processing was used to minimize the effects of irregular ventricular morphology on registration (e.g., either slitlike or mildly dilated ventricles). The first aim of the study was to analyze changes in patients who had been treated with CSF shunting on a chronic basis in comparison with age- and sexmatched healthy control participants. We hypothesized that FA would be reduced in PVWM tracts even in the absence of significant ventricular dilation and that reduced FA would be associated with smaller ventricle size (e.g., slit ventricles). This would be an indication that similar to ventricular dilation, overdrainage can have a deleterious long-term effect on the brain. Our second aim was to investigate the relationship between ventricular volume as well as FA and relevant clinical outcome measures among the patients. Our hypothesis was that reduced FA would be predictive of worse headache symptoms and reduced quality of life (QoL).

\section{Methods \\ Subjects}

Hydrocephalus patients were identified by chart review for participation in the study, based on age at first shunt placement $(<2$ years old) and documented history of headache. Healthy controls, with no history of neurological disorders, were sex and age matched (1:1) to the patients. To account for more significant white matter changes in children, patients younger than 19 years old were age matched within 1 year of age; otherwise, they were age matched within 3 years of age.

Patients identified by chart review were invited to participate in the study by mail and subsequent follow-up phone contact. All patients (or a parent/guardian) filled out an online screening survey consisting of a brief history, including current headache symptoms, shunting history, and potential MRI contraindications. All patients meeting the inclusion criteria were invited to participate, irrespective of current headache symptoms. Because of our hypothesis that the effect of ventricular enlargement on the white matter is different from the effect of chronic shunting and slit ventricles, patients with enlarged ventricles (total ventricle volume $>60 \mathrm{~cm}^{3}$ ) were excluded from the study. The ventricle size cutoff was chosen as twice the upper limit of normal, as determined from an independent sample of 100 control subjects at the Albert Einstein College of Medicine Gruss MR Research Center, and was also the upper limit for which we were able to obtain reliable registration to the TBSS template (see Table 3).

The study protocol was Health Insurance Portability and Accountability Act (HIPAA) compliant and was approved by the Albert Einstein College of Medicine internal review board. Informed consent was obtained from all participants or their parent/guardian.

\section{Patient Surveys}

REDCap electronic data capture tools ${ }^{18}$ were used to collect and retain patient survey information. Patients completed a questionnaire (consisting of 171 questions for adults and 178 for children, with patients 18 years 
and older considered adults); control data were not collected, as the questions were not relevant-all controls were screened for absence of prior neurological disease and history of headache. Clinical data were related to the patients' medical condition (e.g., hydrocephalus etiology, age at first shunt placement, revision history, medication usage, and depression). Functional outcome was assessed with headache and QoL measures. Headache history and severity information included both qualitative and quantitative measures. Qualitative information included responses to questions about variables such as postural effects on headache severity. Quantitative headache scores used the Headache Disability Inventory (HDI), an instrument consisting of 25 questions covering both social/emotional and physical effects of headache on QoL. ${ }^{24}$ HDI scores range from 0 to 100 , with higher scores representing greater impact of headache. Overall QoL was measured with the Hydrocephalus Outcome Questionnaire (HOQ), which represents physical, emotional, and cognitive domains of health-related QoL and has been shown to have excellent test-retest reliability, interrater reliability, and internal consistency. ${ }^{34} \mathrm{HOQ}$ scores range from 0 to 1 , with higher scores indicating better QoL.

\section{MRI Acquisition}

Magnetic resonance imaging was performed using a 3-T Philips scanner (Achieva TX, Philips Medical Systems) with a 32-channel head coil. DTI data were acquired using a single-shot, spin echo planar imaging (EPI) sequence with TE $66 \mathrm{msec}$, TR $10,000 \mathrm{msec}$, voxel size $2 \mathrm{~mm}$ isotropic, max b-factor 800 , and 32 noncollinear directions. B0 field map images (gradient echo, TE 2.4 msec, delta TE $2.3 \mathrm{msec}$, TR $20 \mathrm{msec}$, voxel size $4 \mathrm{~mm}$ isotropic) were collected to correct for EPI-related distortion. High-resolution T1-weighted images (TE $4.6 \mathrm{msec}$, TR $9.9 \mathrm{msec}$, voxel size $1 \mathrm{~mm}$ isotropic) were acquired to provide anatomical images for postprocessing and segmentation of anatomical regions of interest (ROI).

\section{Image Processing}

DTI images were eddy and motion corrected. DTI and T1-weighted high-resolution images were brain extracted using BET from the FSL FMRIB Structural Toolkit. ${ }^{52}$ FA maps were obtained using the dtifit function within the FSL FMRIB Diffusion Toolkit ${ }^{8}$ and distortion corrected using field maps.

Two methods were used to delineate structural ROIs, manual and TBSS-based labeling; manual ROIs were used primarily to validate the automated TBSS method. Six regions were manually traced: the anterior limb of the internal capsule (ALIC), right and left; the posterior limb of the internal capsule (PLIC), right and left; and the genu and splenium of the corpus callosum ( $\mathrm{gCC}$ and sCC, respectively). These were drawn in the axial plane on highresolution T1-weighted images with the aid of FA maps (binarized at FA $>0.3$ to minimize bias) and were verified by a neuroradiologist (A.K.S.) with 5 years of experience in these techniques. Internal capsule ROIs were drawn on a single axial slice, while corpus callosum ROIs covered 8-10 slices (Fig. 1). In both cases, care was taken to

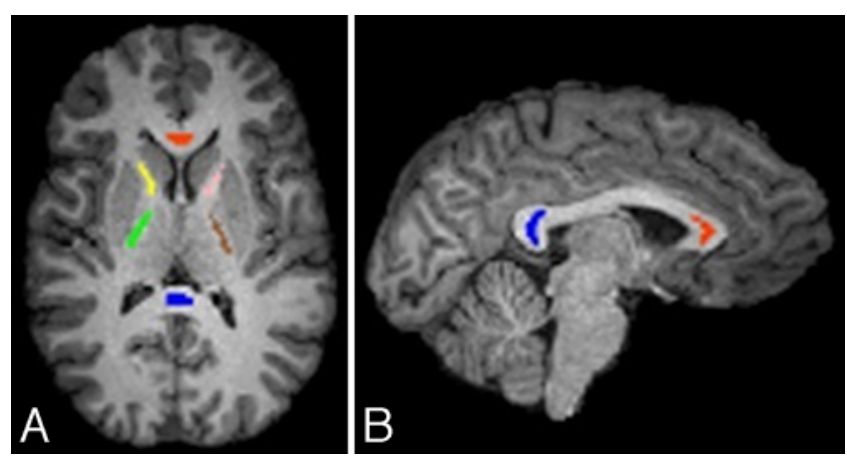

FIG. 1. Manual ROI masks for the corpus callosum and internal capsule, overlaid on axial (A) and sagittal (B) T1-weighted MR images. Figure is available in color online only.

avoid the borders of the structure. The quality of the TBSS registrations, which could be affected by disease-related distortions and bias the measurements, was validated by comparing TBSS-based FA values against the manual ROI FA values (the gold standard), within the 6 manually delineated structures from the manual tracings. As a second check on the registration errors, each individual participant's TBSS skeleton was visually assessed against the study-specific target skeleton and rated for registration quality. Each individual with a visually poor registration of a specific structure, defined as less than approximately $60 \%$ overlap between the subject and target skeleton, was excluded from the statistical analysis for that structure. Structures for which more than 7 patients had poor registration were removed entirely from the statistical analysis.

A custom region-growing algorithm was used to extract total and subregion ventricular volumes. Briefly, CSF was segmented from T1-weighted images using FSL's FAST (FMRIB's Automated Segmentation Tool) ${ }^{66}$ Region growing was then applied using iterative dilation of 3-4 manually placed seed points within the lateral ventricles, manually editing when needed. Regional ventricular volumes were then calculated using cut lines at the interthalamic adhesion (to separate anterior vs posterior horns) and the trigone of the lateral ventricle (to separate posterior vs temporal horns), as well as right-left separation. The Evans ratio and frontal occipital horn ratio $(\mathrm{FOHR})^{41}$ were measured from T1-weighted images. All volumetric measurements were adjusted for total intracranial volume. ${ }^{16,59}$

\section{Tract-Based Spatial Statistics}

Automated segmentation was performed using TBSS within the FSL FMRIB Diffusion Toolkit ${ }^{53}$ in combination with the JHU ICBM-DTI-81 white matter labels atlas ${ }^{40,42}$ for ROI extraction. Briefly, all participants were registered to a target image by nonlinear registration (FNIRT) ${ }^{4}$ to produce a study-specific FA template; the target image was chosen as the FA image for a particular participant, which minimized the average warp field across all participants..$^{53} \mathrm{~A}$ mean FA image (across all participants with FA threshold of 0.2) and a study-specific TBSS FA skeleton were then generated. The FA map for each participant was then projected onto the mean FA skeleton. Voxelwise statistics were calculated using the FSL randomise func- 
tion on the skeletonized DTI parameter maps, ${ }^{62}$ using the threshold free cluster enhancement option and familywise error corrected at $\mathrm{p}<0.05 .^{48}$

Regional DTI measures were extracted using the JHU ICBM-DTI-81 white matter labels atlas. ${ }^{40}$ The atlas was aligned by nonlinear registration of the JHU ICBM-FA-1 $\mathrm{mm}$ template to the study-specific target and application of the warp field to the anatomical labels. The JHU atlas consists of 48 distinct white matter tracts. The resultant study-specific target skeleton, overlaid on the JHU labels, is illustrated in Supplemental Fig. A1. Regional mean DTI parameters (FA, axial diffusivity [AD], etc.) were extracted by multiplying the skeletonized DTI parameter map by the morphed JHU ICBM-DTI-81 white matter labels.

\section{Statistical Analysis}

TBSS-based segmentation results were validated by comparison with the manual segmentation results as the gold standard, using the interclass correlations between the 2 techniques for all subjects in the 6 manually delineated regions. For each region in the JHU atlas, mean FA values (as well as other parameters, e.g., mean diffusivity $[\mathrm{MD}])$ in patients were compared with their age- and sex-matched controls by paired t-test. Paired t-tests were possible because of the 1:1 age and sex matching, where multiple comparisons were corrected with a false discovery rate (FDR) of 0.05 . Ventricular volumes were similarly compared between the 2 groups.

The next part of the analysis was performed within the patient group to assess the associations between FA, ventricular volume, or the clinical outcome measures (headache disability [HDI] and QoL [HOQ], using both overall and individual domain scores) and 3 clinically relevant covariates: age at first shunt surgery (binary: $\leq 3$ vs $>3$ months), number of revisions (binary: $0-1$ vs $\geq 2$ ), and shunt placement (binary: frontal vs occipital). In the first set of models, we assessed the relationship between HDI/HOQ (dependent variable) and a primary predictor of FA or ventricular volume, with and without adjustment for the covariates. Pearson's correlations were computed as the association unadjusted for covariates, and multivariable linear regression models were used to assess whether these associations held after adjustment for the clinically relevant covariates. In the second set of models, we used similar methods to investigate the relationship between FA (dependent variable) and ventricular volume (primary predictor), with and without adjustment for the covariates. All statistical analyses were performed in IBM SPSS version 22.0 (IBM Corp.) and SAS 9.4 (SAS Institute Inc.).

\section{Results}

Thirty-two hydrocephalus patients responded to the survey with interest in participating in the study. Four withdrew prior to imaging, 2 were unable to complete the MRI examination, and 5 were excluded because their total ventricular volume was greater than $60 \mathrm{~cm}^{3}$ (i.e., above the threshold for inclusion). Thus, data were collected from 21 patients, who were then age and sex matched to 21 healthy controls (Table 1). Demographic data for the patients and controls are shown in Table 1.
Table 1 also shows the results for the functional outcome measures in the patient group (HOQ and HDI). The mean HDI score for the patient group indicated moderate impact, although the scores spanned the full range (0-96). In contrast, the mean HOQ score indicated good QoL, with the major impact in cognitive and social-emotional domains. Scores ranged from fair to good, with no patients reporting severe impact on QoL.

TABLE 1. Participant demographics and clinical outcome measures

\begin{tabular}{|c|c|c|}
\hline Characteristic & $\begin{array}{l}\text { Controls } \\
(n=21)\end{array}$ & $\begin{array}{c}\text { Patients } \\
(n=21)\end{array}$ \\
\hline \multicolumn{3}{|l|}{ Age in yrs } \\
\hline Mean (SD) & $22.1(8.0)$ & $22.4(8.3)$ \\
\hline Median & 22 & 19 \\
\hline Range & $8-38$ & $9-39$ \\
\hline \multicolumn{3}{|l|}{ Sex } \\
\hline Male & 8 & 8 \\
\hline Female & 13 & 13 \\
\hline \multicolumn{3}{|l|}{ Race/ethnicity } \\
\hline Hispanic & 0 & 0 \\
\hline Non-Hispanic, white & 11 & 14 \\
\hline Non-Hispanic, black & 4 & 6 \\
\hline Non-Hispanic, other & 6 & 1 \\
\hline Years of education, mean (SD) & $13.6(4.9)$ & $12.0(3.5)$ \\
\hline \multicolumn{3}{|l|}{ Cause of hydrocephalus } \\
\hline $\mathrm{IVH}$ & & 4 \\
\hline Congenital & & 8 \\
\hline Spina bifida & & 3 \\
\hline Meningitis & & 1 \\
\hline Tumor & & 1 \\
\hline Unknown & & 4 \\
\hline Age in mos at first shunt, mean (SD) & & $4.2(4.8)$ \\
\hline \multicolumn{3}{|l|}{ No. of revisions } \\
\hline 0 or 1 & & 9 \\
\hline$\geq 2$ & & 12 \\
\hline Shunt infection & & 8 \\
\hline \multicolumn{3}{|l|}{ Shunt placement } \\
\hline Frontal & & 7 \\
\hline Occipital & & 14 \\
\hline \multicolumn{3}{|l|}{$\mathrm{HOQ}$, mean (SD) } \\
\hline Overall & & $0.75(0.16)$ \\
\hline Physical & & $0.83(0.10)$ \\
\hline Social-emotional & & $0.72(0.21)$ \\
\hline Cognitive & & $0.70(0.22)$ \\
\hline \multicolumn{3}{|l|}{ HDI, mean (SD) } \\
\hline Total & & $36.29(26.72)$ \\
\hline Emotional & & $18.57(13.96)$ \\
\hline Functional & & $17.71(13.26)$ \\
\hline
\end{tabular}

$\mathrm{HDI}=$ Headache Disability Inventory; $\mathrm{HOQ}=$ Hydrocephalus Outcome Questionnaire; IVH = intraventricular hemorrhage.

Values are numbers of patients unless otherwise indicated. 
TABLE 2. FA values for corpus callosum and internal capsule: control and patient group data and paired group comparisons

\begin{tabular}{|c|c|c|c|c|c|c|}
\hline \multirow[b]{2}{*}{ Structure } & \multicolumn{2}{|c|}{ Controls } & \multicolumn{2}{|c|}{ Patients } & \multicolumn{2}{|c|}{ p Value* } \\
\hline & Manual & TBSS & Manual & TBSS & Manual & TBSS \\
\hline Genu & $0.791(0.034)$ & $0.680(0.024)$ & $0.796(0.052)$ & $0.659(0.044)$ & 0.718 & 0.70 \\
\hline Splenium & $0.807(0.050)$ & $0.731(0.021)$ & $0.731(0.092)$ & $0.623(0.096)$ & 0.004 & $<0.001$ \\
\hline PLIC-It & $0.677(0.034)$ & $0.669(0.032)$ & $0.643(0.049)$ & $0.648(0.034)$ & 0.022 & 0.031 \\
\hline PLIC-rt & $0.677(0.034)$ & $0.677(0.023)$ & $0.648(0.058)$ & $0.652(0.041)$ & 0.042 & 0.005 \\
\hline ALIC-It & $0.649(0.053)$ & $0.568(0.034)$ & $0.608(0.055)$ & $0.553(0.044)$ & 0.030 & 0.17 \\
\hline ALIC-rt & $0.616(0.051)$ & $0.560(0.027)$ & $0.614(0.062)$ & $0.559(0.037)$ & 0.90 & 0.97 \\
\hline
\end{tabular}

$A L I C=$ anterior limb of the internal capsule; PLIC = posterior limb of the internal capsule.

Data are presented as mean (SD).

* Controls versus patients.

\section{Imaging Data Quality Assurance}

High-quality DTI images, with no evidence of motion artifact, were obtained in all 42 participants. In 2 patients there was a significant metal artifact (from the shunt in one and from a palate expander in the other) obscuring part of the DTI image field of view. In each of these cases, the images and TBSS results were carefully screened and we verified that none of the white matter tracts analyzed as part of the study were affected by the metal artifact. As noted in Methods, structures for which 7 or more participants had suboptimal registration to the template were excluded from analysis. For this reason, the fornix and the hippocampal portion of the left cingulum were excluded.

\section{Validation of TBSS-Based ROI Method Using Manual ROI Method}

FA values from the 6 manually drawn ROIs were mostly higher than the TBSS-based values; this difference can be expected, since the manual ROIs explicitly excluded the low-FA borders of the structures, while this exclusion cannot be guaranteed with an automated segmentation method. Thus, the 2 methods showed excellent correspondence; the interclass correlation coefficient (ICC) ranged from 0.62 to 0.85 in the gCC, sCC, PLIC, and right ALIC and was somewhat lower in the left ALIC (0.42). The mean FA was significantly lower in patients than in controls $(\mathrm{p}<$ 0.05) in all structures except for the gCC and right ALIC using both methods (Table 2). FA comparisons between patients and controls for the 6 manual ROIs are shown in Fig. 2. The only difference between the 2 methods was in the left ALIC, for which the manual method showed a significantly lower FA in patients (FA in patients for TBSS was also lower, but the difference was not significant).

\section{FA and Ventricular Volume: Patient Versus Control Comparisons}

Ventricular volumes and linear indices (Evans ratio and FOHR) are shown in Table 3. Except for the anterior horn volume, all ventricular volume measurements were significantly larger in patients than in controls. Qualitatively, the posterior horns in patients were normal to mildly dilated, while the anterior horns had a larger range, from slitlike to mildly dilated.

Using the TBSS method, DTI results from 46 struc- tures of the JHU ICBM-DTI-81 white matter labels were extracted. The mean FA values were significantly lower (q $<0.05$ ) for patients than for controls in 20 structures (Fig. 3A). A visual representation of the significant portion of the TBSS white matter skeleton is shown in Fig. 3B. Structures with decreased FA were predominantly posterior and included the body and splenium of the corpus callosum, posterior and retrolenticular limbs of the internal capsule, corona radiata, and cingulum. A complete list of the significant structures and regional FA measures can be found in Supplemental Table A1. Of the 20 structures demonstrating reduced FA, 17 had increased radial diffusivity (RD), while only 5 had increased MD, and 3 had decreased AD (FDR corrected; see Supplemental Table A2). No structures had increased FA in patients compared with controls.

\section{Associations of HOQ/HDI With FA and Ventricular Volume}

FA or ventricular volumes that were significantly correlated with $\mathrm{HOQ} / \mathrm{HDI}$ without covariate adjustment are listed in Table 4. Patients with higher FA in the right retrolenticular limb of the internal capsule (RLIC), right posterior thalamic radiation (PTR), and right tapetum, all structures in the posterior PVWM, had better clinical outcomes. Specifically, positive correlations between HOQ and FA and negative correlations between HDI and FA were found. There was a positive correlation between

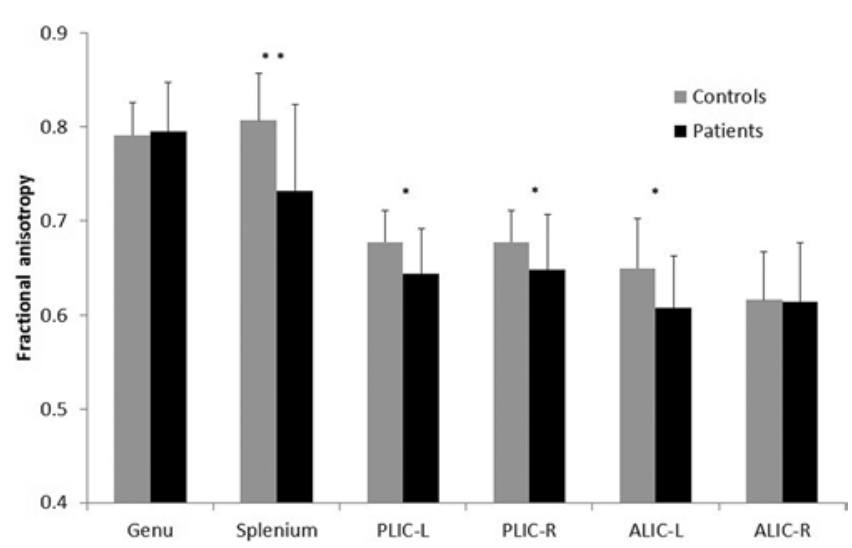

FIG. 2. Group comparisons of FA values within the 6 manually delineated ROls. ${ }^{*} p<0.05 ;{ }^{* *} p<0.01$. 
TABLE 3. Ventricular and subventricular information

\begin{tabular}{|c|c|c|c|}
\hline Variable & Controls & Patients & $p$ Value \\
\hline Total lat ventricle vol & $10.72(4.91)$ & $16.92(10.85)$ & 0.017 \\
\hline \multicolumn{4}{|l|}{ Evans ratio } \\
\hline Total & $0.24(0.03)$ & $0.28(0.05)$ & 0.001 \\
\hline $\mathrm{Lt}$ & $0.23(0.03)$ & $0.28(0.05)$ & 0.001 \\
\hline Rt & $0.25(0.03)$ & $0.28(0.07)$ & 0.051 \\
\hline \multicolumn{4}{|l|}{ FOHR } \\
\hline Total & $0.33(0.03)$ & $0.38(0.06)$ & 0.002 \\
\hline $\mathrm{Lt}$ & $0.33(0.03)$ & $0.38(0.06)$ & 0.001 \\
\hline Rt & $0.33(0.03)$ & $0.37(0.07)$ & 0.009 \\
\hline \multicolumn{4}{|l|}{ Anterior horn vol } \\
\hline Total & $4.84(2.21)$ & $5.38(4.73)$ & 0.626 \\
\hline $\mathrm{Lt}$ & $2.41(1.21)$ & $3.04(2.65)$ & 0.322 \\
\hline Rt & $2.43(1.13)$ & $2.35(2.59)$ & 0.886 \\
\hline \multicolumn{4}{|l|}{ Posterior horn vol } \\
\hline Total & $5.61(3.23)$ & $10.94(6.77)$ & 0.002 \\
\hline $\mathrm{Lt}$ & $2.83(1.81)$ & $5.67(3.70)$ & 0.004 \\
\hline Rt & $2.78(1.52)$ & $5.27(3.66)$ & 0.006 \\
\hline \multicolumn{4}{|l|}{ Temporal horn vol } \\
\hline Total & $0.26(0.16)$ & $0.60(0.69)$ & 0.043 \\
\hline $\mathrm{Lt}$ & $0.11(0.08)$ & $0.37(0.57)$ & 0.055 \\
\hline Rt & $0.15(0.11)$ & $0.23(0.26)$ & 0.215 \\
\hline
\end{tabular}

Data are presented as mean (SD). All volume measures are in cubic centimeters $\left(\mathrm{cm}^{3}\right)$ and corrected for total brain volume.

FA in the left external capsule and HDI (Table 4). With respect to ventricular volume, there was a positive association between left occipital ventricular volume and HOQ and a negative association between left frontal ventricular volume and HDI, both indicating improved outcome with increased ventricular volume.

\section{Associations of FA With Ventricular Volume}

Brain regions with significant correlations without covariate adjustment are listed in Table 5. Larger ventricular volume was associated with higher FA in specific white matter structures, including the posterior and retrolenticular parts of the internal capsule. The only exception to this was the right hippocampal and cingulate gyrus components of the cingulum (right $\mathrm{CGH}$ and right CGC, respectively), in which higher FA was associated with decreased volume.

\section{Discussion}

The goal of this study was to demonstrate alterations in white matter integrity, as indicated by DTI-derived fractional anisotropy (FA), in patients with chronic, shunttreated hydrocephalus compared with healthy controls, and to investigate the relationship between these changes and clinical outcome measures. In a group of patients with slit to mildly dilated ventricles, FA is lower in many white matter tracts, primarily those surrounding the lateral ventricles, although more distal tracts are also affected. These changes are driven by an increase in the radial diffusivity (RD), suggesting demyelination of the PVWM as a potential mechanism. ${ }^{54}$ Furthermore, we have shown that diminished QoL and worse headache symptoms are both indicated by small ventricle size as well as reductions in white matter integrity. It has been hypothesized that CSF shunting early may lead to worse outcome and deleterious effects on the brain, ${ }^{26}$ based on data showing an association between younger age at shunt placement and the occurrence of slit ventricles. We thus used multivariable regression models with clinically relevant covariates (age
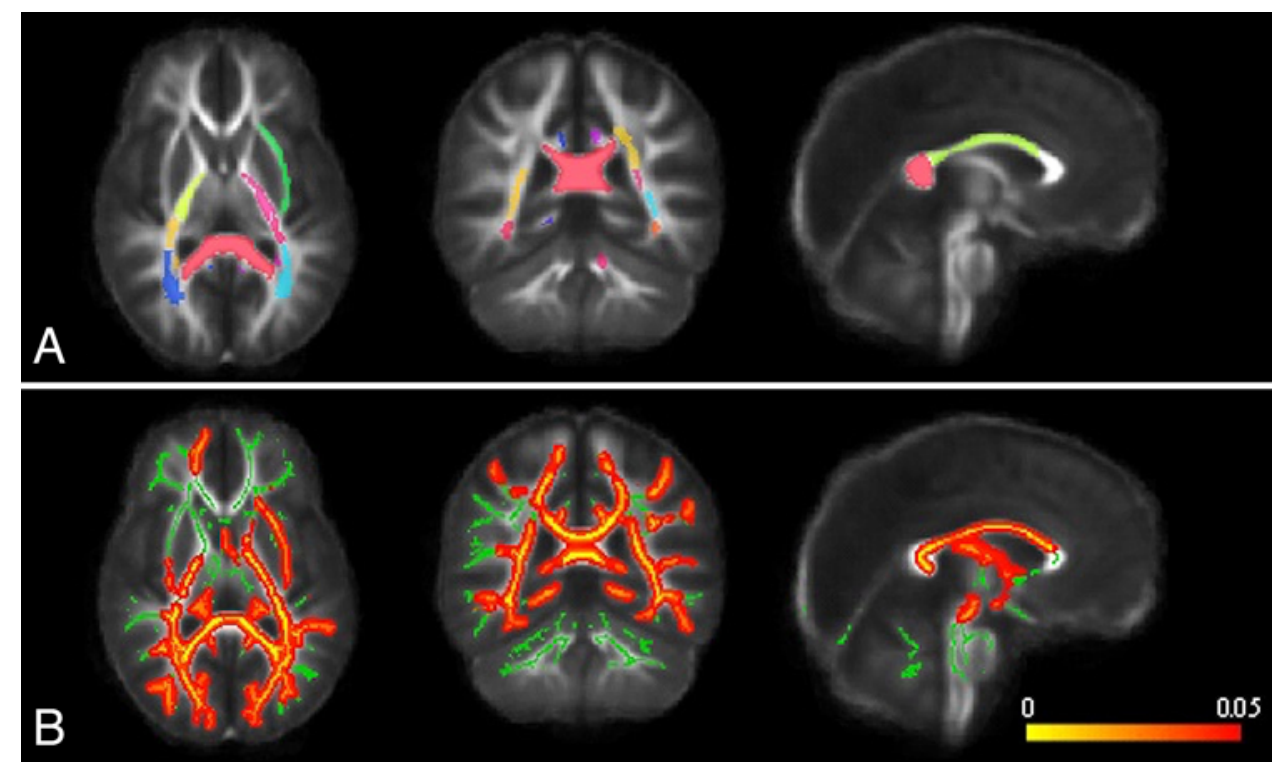

FIG. 3. JHU white matter structures (rainbow, A) and TBSS skeleton mask (green, B) and "thickened" skeleton (red/yellow, B) showing structures where FA patients $<$ FA controls ( $p<0.05$, FWE corrected) overlaid on mean FA map. Figure is available in color online only. 
TABLE 4. Correlations of FA and volume with $H O Q$ and $H D I$ outcome measures

\begin{tabular}{|c|c|c|c|c|}
\hline Dependent Variable & Primary Predictor \& Region & Unadjusted & Adjusted & Significant Covariate \\
\hline \multicolumn{5}{|l|}{$\mathrm{HOQ}$} \\
\hline Overall & FA: CGH-rt & $0.567(p=0.018)$ & $p=0.020$ & - \\
\hline Overall & FA: TAP-rt & $0.455(p=0.038)$ & $p=0.022$ & - \\
\hline Physical & FA: TAP-rt & $0.442(p=0.045)$ & $p=0.002$ & ShP: $0.12 \pm 0.04(0.004)$ \\
\hline Physical & FA: PTR-rt & $0.534(p=0.013)$ & $p=0.13$ & - \\
\hline Physical & FA: RLIC-rt & $0.464(p=0.034)$ & $p=0.069$ & - \\
\hline Social-emotional & FA: TAP-rt & $0.544(p=0.011)$ & $p=0.013$ & - \\
\hline Cognitive & FA: CGH-rt & $0.703(p=0.002)$ & $p=0.010$ & - \\
\hline Cognitive & Vol: occipital-It & $0.475(p=0.030)$ & $p=0.15$ & - \\
\hline \multicolumn{5}{|l|}{ HDI } \\
\hline Total & FA: RLIC-rt & $-0.457(p=0.037)$ & $p=0.12$ & - \\
\hline Total & FA: PTR-rt & $-0.499(p=0.021)$ & $p=0.045$ & - \\
\hline Total & FA: EC-It & $0.453(p=0.039)$ & $p=0.047$ & - \\
\hline Functional & FA: RLIC-rt & $-0.454(p=0.039)$ & $p=0.16$ & - \\
\hline Functional & FA: PTR-rt & $-0.489(p=0.024)$ & $p=0.080$ & - \\
\hline Functional & FA: TAP-rt & $-0.482(p=0.027)$ & $p=0.14$ & - \\
\hline Emotional & FA: RLIC-rt & $-0.444(p=0.044)$ & $p=0.11$ & - \\
\hline Emotional & FA: PTR-rt & $-0.490(p=0.024)$ & $p=0.030$ & - \\
\hline Emotional & FA: TAP-rt & $-0.456(p=0.038)$ & $p=0.11$ & - \\
\hline Emotional & FA: EC-It & $0.453(p=0.039)$ & $p=0.057$ & - \\
\hline Functional & Vol: frontal-It & $-0.441(p=0.045)$ & $p=0.047$ & - \\
\hline
\end{tabular}

$\mathrm{CGH}=$ hippocampal component of cingulum; EC = external capsule; HDI = Headache Disability Inventory; HOQ = Hydrocephalus Outcome Questionnaire; PTR = posterior thalamic radiation; RLIC = retrolenticular internal capsule; $\mathrm{ShP}=$ shunt placement; TAP = tapetum.

Pearson correlations that were significant without adjustment for covariates are listed. Unadjusted correlations are reported as $r(p)$, adjusted correlations are reported as $p$, and covariates are reported as beta \pm SE (p). Correlations that are significant after adjustment are shown in boldface type.

at first shunt, number of revisions, and shunt placement) to test this hypothesis. However, the associations were weak and the direction of the associations was variable, so it is difficult to draw any conclusions from our data about the effect of shunt history on white matter integrity or clinical outcome.

DTI has been widely used in the neuroimaging community over the last 2 decades and has been crucial in documenting changes indicative of white matter injury in a multitude of brain disorders. ${ }^{5,14,32,58}$ Many of these studies have been in cases where there is no radiologically overt alteration in brain morphology, readily allowing image registration to standard brain templates and comparison between patient and control study groups. This becomes more challenging and more readily susceptible to registration errors in the presence of overt brain pathology, such as in hydrocephalus, where ventricular morphology is often severely distorted (even in cases of normal ventricle size, their shape is often distorted from effects such as overdrainage and repeated shunt failure). Thus, the vast majority of DTI studies in hydrocephalus have used manual ROI approaches to obviate this issue. This limits analysis to selected areas simply due to the time-intensive nature of manual ROI tracing and can also introduce operator bias in region selection, since overt ventricular distortion unblinds the operator to patient versus control identification.

In this article, we investigated whole-brain white matter integrity in shunt-dependent hydrocephalus patients using
TBSS. ${ }^{53}$ TBSS is employed in order to improve on issues with standard registration algorithms through 1) carefully tuned nonlinear registration, followed by 2) projection onto an alignment-invariant tract representation (the "mean FA skeleton"). The use of TBSS can improve the sensitivity, objectivity, and interpretability of analysis of multisubject diffusion imaging studies. By including measurements of manually traced ROIs in 2 of the most widely studied PVWM tracts, and through a qualitative scoring scale in other tracts, we verified the robust nature of TBSS registration, even in the presence of morphological distortion of the brain due to ventricular remodeling from chronic hydrocephalus. By combining TBSS and automated ROI analysis, we obtained DTI parameters for each JHU white matter structure, allowing further statistical analyses to be performed. It should be noted that the ALIC is more sensitive to ROI placement due to larger spatial variability in FA; this may explain the discrepancy between the manual and TBSS results in this structure, as well as the left-right difference in the group comparisons (i.e., group differences were only seen in the left ALIC, and not in the right ALIC). In contrast, the posterior limb is more spatially homogeneous in FA values and thus less susceptible to this error.

In contradistinction to many hydrocephalus studies that have found regions of both reduced and increased FA, $, 1,5$, 9,21,30,31 we only found decreases in FA. Furthermore, prior studies have almost exclusively focused on patients with 
TABLE 5. Correlations between ventricular volume and mean FA

\begin{tabular}{|c|c|c|c|c|}
\hline Dependent Variable (FA) & Primary Predictor (vol) & Unadjusted & Adjusted & Significant Covariate \\
\hline PTR-rt & Frontal-total & $0.433(p=0.050)$ & $p=0.30$ & ShP: $0.04 \pm 0.02(0.049)$ \\
\hline PTR-rt & Frontal-rt & $0.462(p=0.035)$ & $p=0.055$ & ShP: $-0.043 \pm 0.018(0.028)$ \\
\hline CGH-rt & Total & $0.622(p=0.008)$ & $p=0.03$ & - \\
\hline CGH-rt & Frontal-total & $0.558(p=0.020)$ & $p=0.043$ & - \\
\hline CGH-rt & Frontal-It & $0.600(p=0.011)$ & $p=0.014$ & ShA: $-0.053 \pm 0.023(0.045)$ \\
\hline CGH-rt & Occipital-total & $0.630(p=0.007)$ & $p=0.026$ & - \\
\hline CGH-rt & Occipital-It & $0.705(p=0.002)$ & $p=0.005$ & - \\
\hline CGH-rt & Occipital-rt & $0.486(p=0.048)$ & $p=0.188$ & - \\
\hline CGH-rt & Temporal-It & $-0.502(p=0.040)$ & $p=0.145$ & - \\
\hline RLIC-rt & Frontal-total & $0.561(p=0.021)$ & $p=0.006$ & - \\
\hline RLIC-rt & Frontal-It & $0.544(p=0.011)$ & $p=0.037$ & - \\
\hline RLIC-rt & Frontal-rt & $0.468(p=0.032)$ & $p=0.051$ & - \\
\hline PLIC-rt & Total & $0.508(p=0.019)$ & $p=0.010$ & - \\
\hline PLIC-rt & Frontal-total & $0.489(p=0.025)$ & $p=0.002$ & $\begin{array}{l}\text { ShR: } 0.031 \pm 0.015(0.049) \\
\text { ShP: } 0.040 \pm 0.016(0.026)\end{array}$ \\
\hline PLIC-rt & Frontal-It & $0.490(p=0.024)$ & $p=0.01$ & $\begin{array}{l}\text { ShR: } 0.032 \pm 0.015(0.048) \\
\text { ShP: } 0.040 \pm 0.016(0.026)\end{array}$ \\
\hline PLIC-rt & Occipital-total & $0.448(p=0.044)$ & $p=0.003$ & - \\
\hline PLIC-rt & Occipital-It & $0.505(p=0.020)$ & $p=0.022$ & - \\
\hline SCR-rt & Total & $0.513(p=0.017)$ & $p=0.016$ & - \\
\hline SCR-rt & Frontal-It & $0.500(p=0.021)$ & $p=0.004$ & - \\
\hline SCR-rt & Occipital-total & $0.495(p=0.033)$ & $p=0.002$ & - \\
\hline SCR-rt & Occipital-It & $0.613(p=0.003)$ & $p=0.005$ & - \\
\hline SCR-It & Occipital-It & $0.458(p=0.02)$ & $p=0.042$ & - \\
\hline CGC-rt & Occipital-total & $-0.534(p=0.022)$ & $p=0.046$ & - \\
\hline
\end{tabular}

CGC = cingulate gyrus component of cingulum; $S C R=$ superior corona radiata; $S h A=$ age at first shunt placement; ShR = shunt revisions.

Pearson correlations that were significant without adjustment for covariates are listed. Unadjusted correlations are reported as $r(p)$, adjusted correlations are reported as $p$, and covariates are reported as beta \pm SE (p). Correlations that are significant after adjustment are shown in boldface type.

moderate to severe ventricular dilation ${ }^{1,9,12,63,65}$ and thus have generally assumed that changes in FA are driven by either compression or stretching of the white matter tracts. Our study is the first to show brain-wide reduction in FA even in the absence of significant ventricular dilation. Increases in FA have generally been demonstrated in the PVWM lateral to the ventricles, hypothesized to be due to compression from the ventricular dilation. Furthermore, a number of studies have shown normalization of increased FA in the PLIC and its associated tracts (i.e., the corticospinal tracts and superior corona radiata) following shunting. ${ }^{25,27,37}$ Our findings of reduced FA in these tracts can be explained in one of 2 ways: 1) the acute compression effects of the hydrocephalus resulting in increased FA mask the effects of white matter injury (i.e., FA reduction), which are subsequently unmasked when the ventricular dilation is reduced, or 2) the white matter injury (as indicated by FA reduction) only manifests later in the disease process, a result of either the pathophysiology of the hydrocephalus or long-term shunting. While we cannot determine whether the FA reductions are a result of long-term CSF shunting or of the hydrocephalus itself (e.g., pathological brain changes associated with the disorder, abnormal ICP, or repeated ventricular expansion from shunt failures), our study nonetheless clearly delineates the areas of white matter injury in long-standing, shunted hydrocephalus. Thus, the unique findings of our study may be due to the timing of the scans relative to onset and treatment: prior studies were done in the acute phase with clear ventricular dilation, whereas our patients all had treated hydrocephalus with small to mildly dilated ventricles. Potential mechanisms leading to the widespread decreases in FA we demonstrated are 1) demyelination or axonal loss prior to shunting (where these effects may be masked in the lateral PVWM by compression effects), 2) injury due to the long-standing hydrocephalus, or 3) repeated injury at the time of shunt failure.

Our study in individuals who have been living with shunts for their entire lives, showing widespread decreases in FA and their relationship to outcome, attempts to fill in the gap in the hydrocephalus literature in this patient population. The majority of studies have been conducted in patients with NPH, which tends to develop much later in life. ${ }^{20,21,23,30,31,38}$ However, NPH and pediatric hydrocephalus are very different disorders; most notably, overdrainage and slit ventricles are extremely uncommon in NPH. In comparison with the NPH literature, research in the area of white matter integrity in pediatric hydrocephalus via 
DTI is scant, focusing on acute hydrocephalus and typically with severe ventriculomegaly.1,12,19,33,55,63 There has only been 1 study in patients with chronic hydrocephalus (secondary to spina bifida), and although the authors only looked at global measures of frontal and parietal white matter, they generally found results in good agreement with ours-reduced FA associated with smaller ventricles driven by increases in RD. ${ }^{60}$

With this study, we have expanded on the existing literature by looking at DTI differences in relation to headache severity and QoL in chronic pediatric hydrocephalus, particularly important elements of the symptomatology in these patients-severe, debilitating headaches are one of the chief complaints that bring patients back into the clinic and cause them to worry about a potential shunt failure ${ }^{10,15,51,57}$ Headaches can be due to a variety of reasons, such as CSF overdrainage, or brief periods of ICP elevation, which can even occur in the presence of a functioning shunt. ${ }^{46}$ Although a number of neurosurgeons have proposed mechanisms for the pathophysiology leading to headache in the setting of chronic shunting, and thus regimented treatment guidelines, ${ }^{35,43,47,49}$ there is still no consensus as to the best treatment plan. ${ }^{2,6}$ Treatment would appear to vary widely between neurosurgeons, although all would appear to agree that avoiding slit ventricles is advisable. It is known that ventricular size is a poor indicator of outcome, and our study suggests that DTI may provide an alternate metric for tailoring treatment to provide optimal long-term outcome: we found that increased FA of select PVWM tracts was associated with improved clinical outcome. Furthermore, our findings suggest that in this population improved white matter tract integrity is associated with increased ventricle size, not smaller ventricles, as has been shown in acute hydrocephalus. While our findings should still be considered preliminary-we only included patients with mild ventricular dilation and thus cannot determine if this relationship is unique to patients with slit to mildly dilated ventricles-they are intriguing and warrant further investigation in a larger study that would include patients with a larger range of ventricular dilation. Based on our results, we can suggest that DTI may play a prognostic role, and serve as a suitable biomarker, in the management of hydrocephalus patients who undergo longterm CSF shunting. Ultimately, noninvasive imaging, such as DTI, may be used in the assessment of white matter development following shunt placement or revision surgery to follow renormalization of white matter tracts in parallel with the reduction in ventricular size.

The study has some limitations that should be mentioned. Although our sample size was comparable to that of many other DTI studies in hydrocephalus, it is still small, and the results should be considered exploratory. An important consideration in our patient population is the distorted ventricular morphology from the long-term hydrocephalus. This can lead to a bias of FA in patients compared with controls, because in brains with more morphological distortion, white matter structures could map into gray matter regions with lower FA. The TBSS method was chosen to minimize these misregistration artifacts. In addition, we compared TBSS ROI results with a select subset of manually drawn ROIs, as well as care- fully inspected all white matter structure registration to exclude any region where the registration may have led to erroneous results. Valid application of TBSS, however, did limit our patient selection to those with at most mild ventricular dilation, so as noted above we cannot extend our findings to patients with significant dilation. Finally, the fact that we only found a marginal relationship between ventricular size and clinical outcome (HOQ/HDI) may be due to the distribution of ventricular sizes in our patients-most of the patients had slit to normal ventricles, with only 4 patients having mildly dilated ventricles. In addition, this may be related to our choice of headache and QoL as clinical outcome measures. Both of these clinical measures are dynamic in nature, sometimes changing from day to day, and we only studied patients at a single time point. To offset this issue, our instruments were designed to summarize the longer-term effects of headache and QoL through inquiry of 4-week effects and have been used successfully in other studies. Moreover, we were able to show some associations between our DTI results and these clinical outcome measures, which support our hypothesis of the relationship between reduced FA in specific white matter tracts and headache and/or QoL outcomes. Finally, an important component of the symptomology of pediatric hydrocephalus is the effect on cognitive function, which was not addressed as part of this study and which might be a better outcome measure to show associations with changes in ventricular size. A larger study with a larger number of patients in the normal to mild dilation range might be needed to predict what an "ideal" ventricular size might be.

\section{Conclusions}

In this study, we have presented evidence that hydrocephalus and chronic shunting are associated with changes in white matter integrity, in both periventricular regions and more distal areas. Headache and QoL-related symptoms were related to changes in FA in some of the PVWM tracts, as well as to changes in ventricle size. These findings are consistent with our assumption that reduced ventricular size is related to poor outcome, the opposite effect of that seen in acute hydrocephalus patients with moderate to severe ventricular dilation. Finally, preliminary data suggest that important characteristics of the hydrocephalus treatment (i.e., shunt history) may result in altered white matter integrity, again supporting the hypothesis that early shunting and multiple shunt revisions can have long-term effects on PVWM tracts.

\section{Acknowledgments}

The Rudi Schulte Research Institute generously provided support for this project. We would like to thank Roman Fleysher and Maria Robles of Albert Einstein College of Medicine for assistance with the DTI processing pipeline and assistance with editing and proofreading the manuscript, respectively.

\section{References}

1. Air EL, Yuan W, Holland SK, Jones BV, Bierbrauer K, Altaye $\mathrm{M}$, et al: Longitudinal comparison of pre- and postoperative 
diffusion tensor imaging parameters in young children with hydrocephalus. J Neurosurg Pediatr 5:385-391, 2010

2. Albright AL: Hydrocephalus shunt practice of experienced pediatric neurosurgeons. Childs Nerv Syst 26:925-929, 2010

3. Albright AL, Tyler-Kabara E: Slit-ventricle syndrome secondary to shunt-induced suture ossification. Neurosurgery 48:764-770, 2001

4. Andersson JLR, Jenkinson M, Smith S: Non-linear registration, aka Spatial normalisation. FMRIB Technial Report TR07JA2. Oxford, UK: FMRIB Centre, 2007 (https://www.fmrib.ox.ac.uk/datasets/techrep/tr07ja2/tr07ja2. pdf) [Accessed August 18, 2017]

5. Assaf Y, Ben-Sira L, Constantini S, Chang LC, Beni-Adani L: Diffusion tensor imaging in hydrocephalus: initial experience. AJNR Am J Neuroradiol 27:1717-1724, 2006

6. Baskin JJ, Manwaring KH, Rekate HL: Ventricular shunt removal: the ultimate treatment of the slit ventricle syndrome. J Neurosurg 88:478-484, 1998

7. Basser PJ, Mattiello J, LeBihan D: MR diffusion tensor spectroscopy and imaging. Biophys J 66:259-267, 1994

8. Behrens TE, Johansen-Berg H, Woolrich MW, Smith SM, Wheeler-Kingshott CA, Boulby PA, et al: Non-invasive mapping of connections between human thalamus and cortex using diffusion imaging. Nat Neurosci 6:750-757, 2003

9. Ben-Sira L, Goder N, Bassan H, Lifshits S, Assaf Y, Constantini S: Clinical benefits of diffusion tensor imaging in hydrocephalus. J Neurosurg Pediatr 16:195-202, 2015

10. Browd SR, Ragel BT, Gottfried ON, Kestle JR: Failure of cerebrospinal fluid shunts: part I: Obstruction and mechanical failure. Pediatr Neurol 34:83-92, 2006

11. Buckley RT, Yuan W, Mangano FT, Phillips JM, Powell S, McKinstry RC, et al: Longitudinal comparison of diffusion tensor imaging parameters and neuropsychological measures following endoscopic third ventriculostomy for hydrocephalus. J Neurosurg Pediatr 9:630-635, 2012

12. Cancelliere A, Mangano FT, Air EL, Jones BV, Altaye M, Rajagopal A, et al: DTI values in key white matter tracts from infancy through adolescence. AJNR Am J Neuroradiol 34:1443-1449, 2013

13. Cherubini A, Péran P, Spoletini I, Di Paola M, Di Iulio F, Hagberg GE, et al: Combined volumetry and DTI in subcortical structures of mild cognitive impairment and Alzheimer's disease patients. J Alzheimers Dis 19:1273-1282, 2010

14. Douaud G, Jbabdi S, Behrens TE, Menke RA, Gass A, Monsch AU, et al: DTI measures in crossing-fibre areas: increased diffusion anisotropy reveals early white matter alteration in MCI and mild Alzheimer's disease. Neuroimage 55:880-890, 2011

15. Drake JM, Kestle JR, Milner R, Cinalli G, Boop F, Piatt J $\mathrm{Jr}$, et al: Randomized trial of cerebrospinal fluid shunt valve design in pediatric hydrocephalus. Neurosurgery 43:294305, 1998

16. Hansen TI, Brezova V, Eikenes L, Håberg A, Vangberg TR: How does the accuracy of intracranial volume measurements affect normalized brain volumes? Sample size estimates based on 966 subjects from the HUNT MRI cohort. AJNR Am J Neuroradiol 36:1450-1456, 2015

17. Harris CA, Resau JH, Hudson EA, West RA, Moon C, McAllister JP II: Mechanical contributions to astrocyte adhesion using a novel in vitro model of catheter obstruction. Exp Neurol 222:204-210, 2010

18. Harris PA, Taylor R, Thielke R, Payne J, Gonzalez N, Conde JG: Research electronic data capture (REDCap) - a metadata-driven methodology and workflow process for providing translational research informatics support. J Biomed Inform 42:377-381, 2009

19. Hasan KM, Eluvathingal TJ, Kramer LA, Ewing-Cobbs L, Dennis M, Fletcher JM: White matter microstructural abnormalities in children with spina bifida myelomeningocele and hydrocephalus: a diffusion tensor tractography study of the association pathways. J Magn Reson Imaging 27:700709,2008

20. Hattingen E, Jurcoane A, Melber J, Blasel S, Zanella FE, Neumann-Haefelin T, et al: Diffusion tensor imaging in patients with adult chronic idiopathic hydrocephalus. Neurosurgery 66:917-924, 2010

21. Hattori T, Ito K, Aoki S, Yuasa T, Sato R, Ishikawa M, et al: White matter alteration in idiopathic normal pressure hydrocephalus: tract-based spatial statistics study. AJNR Am J Neuroradiol 33:97-103, 2012

22. Hermoye L, Saint-Martin C, Cosnard G, Lee SK, Kim J, Nassogne MC, et al: Pediatric diffusion tensor imaging: normal database and observation of the white matter maturation in early childhood. Neuroimage 29:493-504, 2006

23. Hoza D, Vlasák A, Hořínek D, Sameš M, Alfieri A: DTIMRI biomarkers in the search for normal pressure hydrocephalus aetiology: a review. Neurosurg Rev 38:239-244, 2015

24. Jacobson GP, Ramadan NM, Norris L, Newman CW: Headache disability inventory (HDI): short-term test-retest reliability and spouse perceptions. Headache 35:534-539, 1995

25. Kamiya K, Hori M, Irie R, Miyajima M, Nakajima M, Kamagata K, et al: Diffusion imaging of reversible and irreversible microstructural changes within the corticospinal tract in idiopathic normal pressure hydrocephalus. Neuroimage Clin 14:663-671, 2017

26. Kan P, Walker ML, Drake JM, Kestle JR: Predicting slitlike ventricles in children on the basis of baseline characteristics at the time of shunt insertion. J Neurosurg 106 (5 Suppl): 347-349, 2007

27. Kanno S, Saito M, Kashinoura T, Nishio Y, Iizuka O, Kikuchi H, et al: A change in brain white matter after shunt surgery in idiopathic normal pressure hydrocephalus: a tractbased spatial statistics study. Fluids Barriers CNS 14:1, 2017

28. Kantarci K, Avula R, Senjem ML, Samikoglu AR, Zhang B, Weigand SD, et al: Dementia with Lewy bodies and Alzheimer disease: neurodegenerative patterns characterized by DTI. Neurology 74:1814-1821, 2010

29. Kestle J, Drake J, Milner R, Sainte-Rose C, Cinalli G, Boop F, et al: Long-term follow-up data from the Shunt Design Trial. Pediatr Neurosurg 33:230-236, 2000

30. Kim MJ, Seo SW, Lee KM, Kim ST, Lee JI, Nam DH, et al: Differential diagnosis of idiopathic normal pressure hydrocephalus from other dementias using diffusion tensor imaging. AJNR Am J Neuroradiol 32:1496-1503, 2011

31. Koyama T, Marumoto K, Domen K, Miyake H: White matter characteristics of idiopathic normal pressure hydrocephalus: a diffusion tensor tract-based spatial statistic study. Neurol Med Chir (Tokyo) 53:601-608, 2013

32. Kraus MF, Susmaras T, Caughlin BP, Walker CJ, Sweeney JA, Little DM: White matter integrity and cognition in chronic traumatic brain injury: a diffusion tensor imaging study. Brain 130:2508-2519, 2007

33. Kulkarni AV, Donnelly R, Mabbott DJ, Widjaja E: Relationship between ventricular size, white matter injury, and neurocognition in children with stable, treated hydrocephalus. J Neurosurg Pediatr 16:267-274, 2015

34. Kulkarni AV, Rabin D, Drake JM: An instrument to measure the health status in children with hydrocephalus: the Hydrocephalus Outcome Questionnaire. J Neurosurg 101 (2 Suppl):134-140, 2004

35. Le H, Yamini B, Frim DM: Lumboperitoneal shunting as a treatment for slit ventricle syndrome. Pediatr Neurosurg 36:178-182, 2002 
36. Le Bihan D, Mangin JF, Poupon C, Clark CA, Pappata S, Molko N, et al: Diffusion tensor imaging: concepts and applications. J Magn Reson Imaging 13:534-546, 2001

37. Mangano FT, Altaye M, McKinstry RC, Shimony JS, Powell SK, Phillips JM, et al: Diffusion tensor imaging study of pediatric patients with congenital hydrocephalus: 1-year postsurgical outcomes. J Neurosurg Pediatr 18:306-319, 2016

38. Marumoto K, Koyama T, Hosomi M, Kodama N, Miyake H, Domen K: Diffusion tensor imaging in elderly patients with idiopathic normal pressure hydrocephalus or Parkinson's disease: diagnosis of gait abnormalities. Fluids Barriers CNS 9:20, 2012

39. Menke RA, Scholz J, Miller KL, Deoni S, Jbabdi S, Matthews PM, et al: MRI characteristics of the substantia nigra in Parkinson's disease: a combined quantitative T1 and DTI study. Neuroimage 47:435-441, 2009

40. Mori S, Oishi K, Jiang H, Jiang L, Li X, Akhter K, et al: Stereotaxic white matter atlas based on diffusion tensor imaging in an ICBM template. Neuroimage 40:570-582, 2008

41. O'Hayon BB, Drake JM, Ossip MG, Tuli S, Clarke M: Frontal and occipital horn ratio: a linear estimate of ventricular size for multiple imaging modalities in pediatric hydrocephalus. Pediatr Neurosurg 29:245-249, 1998

42. Oishi K, Zilles K, Amunts K, Faria A, Jiang H, Li X, et al: Human brain white matter atlas: identification and assignment of common anatomical structures in superficial white matter. Neuroimage 43:447-457, 2008

43. Peraio S, Calcagni ML, Mattoli MV, Marziali G, DE Bonis P, Pompucci A, et al: Decompressive craniectomy and hydrocephalus: proposal of a therapeutic flow-chart. J Neurosurg Sci [epub ahead of print], 2016

44. Rajagopal A, Shimony JS, McKinstry RC, Altaye M, Maloney T, Mangano FT, et al: White matter microstructural abnormality in children with hydrocephalus detected by probabilistic diffusion tractography. AJNR Am J Neuroradiol 34:2379-2385, 2013

45. Reddy GK, Bollam P, Caldito G: Long-term outcomes of ventriculoperitoneal shunt surgery in patients with hydrocephalus. World Neurosurg 81:404-410, 2014

46. Rekate HL: Shunt-related headaches: the slit ventricle syndromes. Childs Nerv Syst 24:423-430, 2008

47. Rekate HL, Kranz D: Headaches in patients with shunts. Semin Pediatr Neurol 16:27-30, 2009

48. Salimi-Khorshidi G, Smith SM, Nichols TE: Adjusting the effect of nonstationarity in cluster-based and TFCE inference. Neuroimage 54:2006-2019, 2011

49. Sandler AL, Goodrich JT, Daniels LB III, Biswas A, Abbott $\mathrm{R}$ : Craniocerebral disproportion: a topical review and proposal toward a new definition, diagnosis, and treatment protocol. Childs Nerv Syst 29:1997-2010, 2013

50. Scheel M, Diekhoff T, Sprung C, Hoffmann KT: Diffusion tensor imaging in hydrocephalus--findings before and after shunt surgery. Acta Neurochir (Wien) 154:1699-1706, 2012

51. Shah SS, Hall M, Slonim AD, Hornig GW, Berry JG, Sharma $\mathrm{V}$ : A multicenter study of factors influencing cerebrospinal fluid shunt survival in infants and children. Neurosurgery 62:1095-1103, 2008

52. Smith SM: Fast robust automated brain extraction. Hum Brain Mapp 17:143-155, 2002

53. Smith SM, Jenkinson M, Johansen-Berg H, Rueckert D, Nichols TE, Mackay CE, et al: Tract-based spatial statistics: voxelwise analysis of multi-subject diffusion data. Neuroimage 31:1487-1505, 2006

54. Song SK, Sun SW, Ju WK, Lin SJ, Cross AH, Neufeld AH: Diffusion tensor imaging detects and differentiates axon and myelin degeneration in mouse optic nerve after retinal ischemia. Neuroimage 20:1714-1722, 2003
55. Sun M, Yuan W, Hertzler DA, Cancelliere A, Altaye M, Mangano FT: Diffusion tensor imaging findings in young children with benign external hydrocephalus differ from the normal population. Childs Nerv Syst 28:199-208, 2012

56. Tuli S, Drake J, Lawless J, Wigg M, Lamberti-Pasculli M: Risk factors for repeated cerebrospinal shunt failures in pediatric patients with hydrocephalus. J Neurosurg 92:3138,2000

57. Tuli S, O'Hayon B, Drake J, Clarke M, Kestle J: Change in ventricular size and effect of ventricular catheter placement in pediatric patients with shunted hydrocephalus. Neurosurgery 45:1329-1335, 1999

58. Werring DJ, Clark CA, Barker GJ, Thompson AJ, Miller DH: Diffusion tensor imaging of lesions and normal-appearing white matter in multiple sclerosis. Neurology 52:1626-1632, 1999

59. Whitwell JL, Crum WR, Watt HC, Fox NC: Normalization of cerebral volumes by use of intracranial volume: implications for longitudinal quantitative MR imaging. AJNR Am J Neuroradiol 22:1483-1489, 2001

60. Williams VJ, Juranek J, Stuebing K, Cirino PT, Dennis M, Fletcher JM: Examination of frontal and parietal tectocortical attention pathways in spina bifida meningomyelocele using probabilistic diffusion tractography. Brain Connect 3:512-522, 2013

61. Williams VJ, Juranek J, Stuebing KK, Cirino PT, Dennis M, Bowman RM, et al: Postshunt lateral ventricular volume, white matter integrity, and intellectual outcomes in spina bifida and hydrocephalus. J Neurosurg Pediatr 15:410-419, 2015

62. Winkler AM, Ridgway GR, Webster MA, Smith SM, Nichols TE: Permutation inference for the general linear model. Neuroimage 92:381-397, 2014

63. Yuan W, Deren KE, McAllister JP II, Holland SK, Lindquist DM, Cancelliere A, et al: Diffusion tensor imaging correlates with cytopathology in a rat model of neonatal hydrocephalus. Cerebrospinal Fluid Res 7:19, 2010

64. Yuan W, Holland SK, Shimony JS, Altaye M, Mangano FT, Limbrick DD, et al: Abnormal structural connectivity in the brain networks of children with hydrocephalus. Neuroimage Clin 8:483-492, 2015

65. Yuan W, Mangano FT, Air EL, Holland SK, Jones BV, Altaye M, et al: Anisotropic diffusion properties in infants with hydrocephalus: a diffusion tensor imaging study. AJNR Am J Neuroradiol 30:1792-1798, 2009

66. Zhang Y, Brady M, Smith S: Segmentation of brain MR images through a hidden Markov random field model and the expectation-maximization algorithm. IEEE Trans Med Imaging 20:45-57, 2001

67. Zheng Z, Shemmassian S, Wijekoon C, Kim W, Bookheimer SY, Pouratian N: DTI correlates of distinct cognitive impairments in Parkinson's disease. Hum Brain Mapp 35:1325-1333, 2014

\section{Disclosures}

The authors report no conflict of interest concerning the materials or methods used in this study or the findings specified in this paper.

\section{Author Contributions}

Conception and design: Wagshul, Tan. Acquisition of data: Wagshul, Tan, Sandler. Analysis and interpretation of data: Wagshul, Tan, Meiri, Mowrey, Suri. Drafting the article: Wagshul, Tan. Critically revising the article: Wagshul, Tan, Meiri, Abbott, Goodrich, Sandler, Suri, Lipton. Reviewed submitted version of manuscript: Wagshul, Tan, Meiri, Abbott, Goodrich, Sandler, Suri, Lipton. Approved the final version of the manuscript on behalf of all authors: Wagshul. Statistical analysis: Tan, Mowrey. 
Administrative/technical/material support: Tan, Meiri. Study supervision: Wagshul.

\section{Supplemental Information}

\section{Online-Only Content}

Supplemental material is available with the online version of the article.

Supplemental Figure and Tables. https://thejns.org/doi/suppl/ 10.3171/2017.6.JNS162784.

\section{Previous Presentations}

This work was presented in part at the 2015 meeting of the International Society for Hydrocephalus and Cerebrospinal Fluid Disorders in Calgary, Alberta, Canada, September 19-21.

\section{Correspondence}

Mark E. Wagshul: Gruss Magnetic Resonance Research Center, Albert Einstein College of Medicine, Bronx, NY. mark.wagshul@ einstein.yu.edu. 